REVESCO. Revista de Estudios Cooperativos

ISSN: $1885-8031$

http://dx.doi.org/10.5209/REVE.57061

\title{
Reestructuración de las cajas de ahorros: evolución del número de oficinas 1986-2013
}

\author{
Cristina Bernad Morcate ${ }^{1}$
}

Recibido: 8 de julio de 2016 / Aceptado: 2 de junio de 2017

Resumen. En este trabajo se analiza el proceso de reestructuración experimentado por las cajas de ahorros españolas durante el periodo 1986 a 2013, a partir de la evolución en su red de oficinas. Con la liberalización que tuvieron a partir de 1989 y la posibilidad de expandirse sin restricciones, se produce un primer periodo de expansión y apertura de oficinas. La crisis económica y financiera, que ha afectado a todo el sector bancario, ha sido más intensa en las cajas de ahorros que se vieron inmersas en un proceso de fusiones, absorciones y a través de Sistemas Institucionales de protección (SIP) que tuvo como consecuencia, entre otras, el cierre de un elevado número de oficinas. Además, el trabajo realiza un análisis a fondo de cada entidad a nivel provincial, destacando el cálculo del índice de Herfindahl para conocer el grado de rivalidad en cada mercado. Finalmente se explica la situación del nuevo escenario tras el proceso de reestructuración.

Palabras clave: Cajas de Ahorro; Oficinas; Regulación.

Claves Econlit: G21.

\section{[en] Reestructuring of savings banks: evolution number of branches 1986- 2013}

Abstract. This paper analyzes the restructuring process experienced by the Spanish savings banks during the period 1986 to 2013, based on the evolution in their network of branches. With the liberalization they had since 1989 and the possibility of expanding without restrictions, there is a first period of expansion and opening of branches. The economic and financial crisis, which has affected the entire banking sector, has been more intense in the savings banks that were involved in a process of mergers and through Institutional Systems of Protection (SIP) the closure of a large number of offices. In addition, the paper performs a depth analysis of each entity at the provincial level, highlighting the calculation of the Herfindahl index to know the degree of rivalry in each market. Finally the situation of the new scenario is explained after the restructuring process.

Keywords: Savings banks; Branches; Regulation.

Sumario. 1. Introducción. 2. Evolución de la red de oficinas de las Cajas de Ahorros en España. 3. Grado de rivalidad. 4. Patrón de comportamiento de las Cajas de Ahorros. 5. Conclusiones. 6. Referencias bibliográficas.

1 Universidad de Zaragoza, España

Dirección de correo electrónico: bernade@unizar.es 
Cómo citar: Bernad Morcate, C. (2017) Reestructuración de las cajas de ahorros: evolución del número de oficinas 1986-2013. REVESCO. Revista de Estudios Cooperativos, No 125, pp. 7-23. DOI: 10.5209/REVE.57061.

\section{Introducción}

La actual crisis financiera ha modificado el panorama del sector financiero español, y en particular a las entidades de crédito de la Economía Social: cajas de ahorros y cooperativas de crédito. Hasta el año 2008, las cajas de ahorros experimentaron un importante sobredimensionamiento que se tradujo en un incremento del número de oficinas bancarias en todo el territorio nacional. Sin embargo, desde ese momento, tuvo lugar un proceso de reestructuración del sector, que afectó, fundamentalmente a las cajas de ahorros. Estas comenzaron un importante proceso de concentración, las cajas pasaron de 45 a 15 en el año 2013 (González y González, 2012) ${ }^{2}$.

En este trabajo vamos a analizar la evolución que han seguido las cajas de ahorros españolas durante el período 1986-2013. Hay que tener en cuenta que desde hace treinta años se han producido importantes acontecimientos (cambios sustanciales en la legislación financiera, una amplia liberalización, tanto desde el punto de vista geográfico como operativo) que han repercutido, fundamentalmente, en la dimensión de la red de oficinas de las cajas de ahorros dado el peso de la banca minorista en nuestro país (Bernad, Fuentelsaz y Gómez. 2005; Calvo, Parejo, Rodríguez y Cuervo, 2010) y en su singularidad, lo que dio lugar a un periodo de expansión.

El objetivo de este trabajo es analizar y profundizar en la transformación que ha sufrido el sistema financiero español durante los últimos 28 años.

Con el fin de conseguir el objetivo apuntado, el trabajo se estructura como sigue. En primer lugar, se analiza la evolución de la red de oficinas de las cajas de ahorros entre 1986 y 2013, En segundo lugar, realizaremos un análisis de la expansión territorial por provincias y por entidades para ver similitudes o diferencias entre unos mercados geográficos u otros o entre unas entidades y otras. Posteriormente, se determinará el grado de rivalidad, mediante el cálculo del índice de Herfindahl, que mide el grado de rivalidad entre cajas de ahorros y se expondrán las consecuencias principales del proceso de reestructuración. También, se analizará el patrón de comportamiento de las cajas de ahorros, a partir del número de oficinas existentes en 2013 para cada una de las quince entidades existentes en la actualidad, profundizando en las consecuencias principales del proceso de reestructuración como el cierre de sucursales. Por último, el trabajo se cierra con las conclusiones que se derivan de él.

2 A 31 de diciembre de 2015, las entidades resultantes de este proceso de restructuración eran: Caixabank, Bankia, Ibercaja Banco, Catalunya Bank, Abanca Corporación Bancaria, KutxaBank, Banco Mare Nostrum, Unicaja Banco, Liberbank, Caixa Ontinyent y Caixa Pollenca (Anuario Estadístico CECA, 2015). 


\section{Evolución de la red de oficinas de las Cajas de Ahorros en España ${ }^{3}$}

Las cajas de ahorros tuvieron plena libertad de operar en todo el territorio nacional a partir de 1989. Por ello resulta interesante ver cómo han evolucionado estas entidades en el transcurso de los años con el objetivo de profundizar en los fenómenos que han marcado la evolución del sector durante los últimos años, es decir, la expansión de las entidades y su posterior reducción.

El cuadro 1 muestra la evolución en el número de oficinas de las cajas de ahorros entre los años 1986-2014. En el año 1986 en España había 11.295 ofícinas, y al final, en 2013, pasaron a haber 15.306 oficinas. Sin embargo, si observamos la tabla vemos que a lo largo de los años no se ha producido un crecimiento paulatino y constante, sino que aumentó la red de oficinas durante unos años y se redujo posteriormente. El máximo de oficinas lo encontramos en el 2008 con 25.035. Años anteriores se había producido un incremento a lo largo de todos los años en los que el máximo crecimiento se da entre los años 1996-2000 con un incremento porcentual entorno al 5\% cada año. Esta situación cambia drásticamente en 2009. Se inicia un periodo en el que se empiezan a cerrar un elevado número de oficinas que será más fuerte en los últimos años (2011-2013) con una reducción porcentual superior al $10 \%$ cada año.

Cuadro. 1. Evolución del número de oficinas de las cajas de ahorros, 1986-20134

\begin{tabular}{|c|c|c|c|c|c|c|c|}
\hline $\mathbf{A} \tilde{\mathbf{N O}}$ & $\mathbf{N}^{\mathbf{0}}$ oficinas & Incremento & \% Variación & AÑ & $\begin{array}{c}\mathbf{N}^{\mathbf{O}} \\
\text { oficinas }\end{array}$ & Incremento & $\begin{array}{c}\text { \% } \\
\text { Variación }\end{array}$ \\
\hline $\mathbf{1 9 8 6}$ & 11.295 & & & $\mathbf{2 0 0 0}$ & 19.297 & 947 & $5,16 \%$ \\
$\mathbf{1 9 8 7}$ & 11.710 & 415 & $3,67 \%$ & $\mathbf{2 0 0 1}$ & 19.842 & 545 & $2,82 \%$ \\
$\mathbf{1 9 8 8}$ & 12.299 & 589 & $5,03 \%$ & $\mathbf{2 0 0 2}$ & 20.349 & 507 & $2,56 \%$ \\
$\mathbf{1 9 8 9}$ & 13.140 & 841 & $6,84 \%$ & $\mathbf{2 0 0 3}$ & 20.893 & 544 & $2,67 \%$ \\
$\mathbf{1 9 9 0}$ & 13.685 & 545 & $4,15 \%$ & $\mathbf{2 0 0 4}$ & 21.529 & 636 & $3,04 \%$ \\
$\mathbf{1 9 9 1}$ & 13.858 & 173 & $1,26 \%$ & $\mathbf{2 0 0 5}$ & 22.443 & 914 & $4,25 \%$ \\
$\mathbf{1 9 9 2}$ & 14.121 & 263 & $1,90 \%$ & $\mathbf{2 0 0 6}$ & 23.457 & 1.014 & $4,52 \%$ \\
$\mathbf{1 9 9 3}$ & 14.264 & 143 & $1,01 \%$ & $\mathbf{2 0 0 7}$ & 24.637 & 1.180 & $5,03 \%$ \\
$\mathbf{1 9 9 4}$ & 14.595 & 331 & $2,32 \%$ & $\mathbf{2 0 0 8}$ & 25.035 & 398 & $1,62 \%$ \\
$\mathbf{1 9 9 5}$ & 15.010 & 415 & $2,84 \%$ & $\mathbf{2 0 0 9}$ & 24.252 & -783 & $-3,13 \%$ \\
$\mathbf{1 9 9 6}$ & 15.874 & 864 & $5,76 \%$ & $\mathbf{2 0 1 0}$ & 23.253 & -999 & $-4,12 \%$
\end{tabular}

3 López González (2010) justifica el estudio de las cajas de ahorros ya que el crecimiento acumulado de la red de oficinas de las cajas de ahorros supera al de la banca poniéndose de manifiesto la mayor capacidad de crecimiento de las primeras propiciada por sus particularidades jurídicas, que les permite canalizar una porción mayor de los beneficios hacia el crecimiento de la red de oficinas (Marbella, 2006), por una mayor especialización en la banca minorista (Coello, 1994), por las repercusiones de la liberalización geográfica de estas entidades (Bernad y otros, 2005; Illueca y otros, 2005).

4 El número de oficinas en 2014 y 2015, es, respectivamente de 14.663 y 14.270 lo que constata el proceso de reducción y cierre. 


\begin{tabular}{|l|l|l|l|l|l|l|l|}
$\mathbf{1 9 9 7}$ & 16.647 & 773 & $4,87 \%$ & $\mathbf{2 0 1 1}$ & 20.719 & -2.534 & $-10,90 \%$ \\
$\mathbf{1 9 9 8}$ & 17.468 & 821 & $4,93 \%$ & $\mathbf{2 0 1 2}$ & 18.409 & -2.310 & $-11,15 \%$ \\
$\mathbf{1 9 9 9}$ & 18.350 & 882 & $5,05 \%$ & $\mathbf{2 0 1 3}$ & 15.306 & -3.103 & $-16,86 \%$ \\
\hline
\end{tabular}

Fuente: Elaboración propia con datos de la CECA

Sin embargo, si observamos en el cuadro 2 la evolución en el número de entidades de cajas de ahorros (incluidas las entidades bancarias que surgen tras el traspaso de la actividad de estas) podemos detectar que este aumento que se ha producido en el número de oficinas no se corresponde con un aumento en el número de entidades, sino al contrario. Con el trascurso de los años se ha pasado de 76 entidades en 1986 a 15 en 2013 (una reducción del 80\%). Los años en los que más se ha reducido esta cifra son en 1990 como consecuencia de la ola de fusiones que se dieron, y en 2010 tras los efectos de la crisis.

Cuadro. 2. Evolución en el número de entidades de cajas de ahorros (1986-2013)

\begin{tabular}{|c|c|c|c|c|c|c|c|}
\hline AÑO & $\begin{array}{l}\mathrm{N}^{0} \text { cajas } \\
\text { ahorros* }\end{array}$ & AÑO & $\begin{array}{l}\mathrm{N}^{\circ} \text { cajas } \\
\text { ahorros * }\end{array}$ & AÑNO & $\begin{array}{l}\mathrm{N}^{\mathbf{0}} \text { cajas } \\
\text { ahorros * }\end{array}$ & AÑO & $\begin{array}{l}\mathbf{N}^{0} \text { cajas } \\
\text { ahorros * }\end{array}$ \\
\hline 1986 & 76 & 1993 & 51 & 2000 & 47 & 2007 & 45 \\
\hline 1987 & 76 & 1994 & 51 & 2001 & 46 & 2008 & 45 \\
\hline 1988 & 76 & 1995 & 50 & 2002 & 46 & 2009 & 45 \\
\hline 1989 & 76 & 1996 & 50 & 2003 & 46 & 2010 & 36 \\
\hline 1990 & 64 & 1997 & 50 & 2004 & 46 & 2011 & 20 \\
\hline 1991 & 56 & 1998 & 50 & 2005 & 46 & 2012 & 15 \\
\hline 1992 & 53 & 1999 & 49 & 2006 & 45 & 2013 & 15 \\
\hline
\end{tabular}

Fuente: Elaboración propia

* incluyendo las que se transforman en fundaciones de otro tipo como bancos

En el primer periodo, de 1986 a 2008, se produce una clara expansión fruto de la amplia liberalización a partir del momento en que las cajas empezaron a disfrutar de libertad de establecimiento por todo el territorio nacional (Illueca, Pastor y Tortosa Ausina. 2005; Bernad, Fuentelsaz y Gómez, 2005; Ibarrondo y Sánchez, 2005; Gutierrez, Palomo y Romero, 2012; Gutierrez, Fernández y Palomo, 2016). Así, en todos los años de este periodo se produce un aumento en el número de oficinas de las cajas de ahorros, más acusado e intenso en los últimos años. Se pueden identificar cuatro subperiodos dentro del mismo. El primero de ellos va desde el año 1986 a 1990. Aunque la eliminación de las restricciones que impedían operar fuera de la Comunidad Autónoma en la que se ubicaba la sede social de la caja de ahorro no tiene lugar hasta 1989, años antes ya se había producido estrategias de crecimiento por parte de algunas entidades dentro de su propia 
comunidad, lo que hace que se produzca una fuerte expansión de la red, en torno al 20 por ciento en este periodo. El segundo va del año 1990 a 1996. En este periodo, el incremento en el número de oficinas se ve reducido ligeramente debido principalmente al cierre de entidades que se da consecuencia de la reestructuración derivada de la ola de fusiones que se produce entre los años 1990 y 1992 ( se pasaron de 76 entidades a 53 en 1992). El tercero comprende los años 1996 al 2008. De nuevo se produce una importante apertura del sector con más de 9.600 nuevas oficinas desde entonces, fruto de la bonanza económica del país. En este destacan los años 2006 y 2007 donde el incremento de oficinas alcanza el máximo de todos los años, superando las 1000 oficinas cada año. El cuarto y último lo constituye el año 2008, cuando el incremento en el número de oficinas vuelve a reducirse sustancialmente (398 nuevas oficinas) fruto de las fusiones que se da entre entidades de crédito, de manera que en este año se reducen las cajas de ahorros a 45.

El segundo periodo comprende los años 2009 a 2013 y se caracteriza por la reducción que se da en el número de oficinas de las cajas de ahorros y en el número de entidades pasaron de 45 a 15) fruto del proceso de reestructuración producido por la crisis financiera.

La Ley del FROB impulsó un intenso proceso de integraciones y fusiones entre cajas de ahorros, lo que modificó radicalmente el mapa de este sector en España. De esta manera entre los años 2009-2010 se produce una reducción en el número de oficinas de más de 1.700, representando aproximadamente un descenso del 7\% de la cifra que había anteriormente en el 2008. Por último, se puede apreciar que en los últimos años reflejados, 2011-2013, existe una drástica reducción en el número de oficinas fruto de esta reestructuración del sector, acabando con casi 8.000 oficinas existentes, lo que representa un decremento de casi un $40 \%$ en tan sólo 3 años.

\section{Grado de rivalidad}

Una vez analizada la expansión territorial puede resultar de interés conocer cómo ha afectado la conducta de las empresas a esta nueva configuración del mercado, es decir, como se ha visto modificado el grado de rivalidad del sector bancario español. Para ello se ha calculado el índice de Herfindahl que mide el grado de concentración general de las cajas de ahorros a partir de las oficinas situadas en cada provincia (Berges, 2003; Palomo y González, 2004; Palomo y Sanchís, 2008; Gutierrez, Palomo y Fernández, 2013; Maudos, 2016).

En el cuadro 3 se muestran los resultados de calcular los índices de Herfindahl para las cajas de ahorros a partir de las oficinas situadas en las 52 provincias. Los datos que aparecen en el mismo permiten ver la evolución de la estructura de los mercados a lo largo del tiempo. Como se puede observar en la mayoría de los mercados provinciales se reduce el nivel de concentración: de las 52 provincias, en 38 disminuye el índice de Herfindahl. Dicha reducción de la concentración se produce a pesar de las fusiones y adquisiciones que tienen lugar durante el periodo y que deberían haber compensado, al menos en parte, el efecto de la expansión en nuevos mercados. 
En el año 1986 nos encontramos las cifras más altas, lo que significa que había una mayor concentración en el sector, o lo que es lo mismo, una menor rivalidad entre las empresas. Así, los unos que aparecen en Asturias, Cantabria, León, Salamanca, Segovia, Soria y Tenerife significan que en estas tierras únicamente existía 1 caja de ahorros en este año, por lo que contaba con poder de monopolio. También merece mención la diferencia de concentración que existía entre Barcelona y Madrid, la primera poco concentrada y la segunda bastante concentrada, que se irían igualando con el paso de los años.

Entre los mercados más concentrados y, por lo tanto, que deberían mostrar menores niveles de rivalidad en el año 2013, destacan las provincias de Tenerife, Navarra, Teruel, Orense, Cuenca y Segovia. Por el contrario, en el otro extremo se sitúa Jaén, Ciudad Real, Alicante, Madrid y Valencia en los que el número equivalente de empresas se sitúa alrededor de $4 .^{5}$

Si observamos lo que ocurre a partir del 2008, vemos que en este año suele estar el pico inferior de concentración en casi todos los mercados provinciales, y posteriormente tiende a aumentar. Esto es así por el gran número de fusiones que se da en estos años que hace que vaya disminuyendo la rivalidad de las empresas al haber menos cajas de ahorros.

Hay que destacar como afirmaron Fuentelsaz, Gómez y Polo (2002) que el grado de rivalidad de los mercados no solo está afectado por el número de cajas de ahorros que compiten en estos mercados, sino que también influye la competencia potencial, lo que significa que el incremento en el número de entidades competidoras en mercados cercanos debería suponer un importante elemento a tener en cuenta en la evaluación de dicha rivalidad.

Cuadro. 3. Índice de concentración de Herfindahl por provincia, 1986-2013.

\begin{tabular}{|c|cccccc|ccccc|}
\cline { 2 - 9 } \multicolumn{1}{c|}{} & $\mathbf{1 9 8 6}$ & $\mathbf{1 9 9 0}$ & $\mathbf{1 9 9 5}$ & $\mathbf{2 0 0 0}$ & $\mathbf{2 0 0 5}$ & $\mathbf{2 0 0 8}$ & $\mathbf{2 0 0 9}$ & $\mathbf{2 0 1 0}$ & $\mathbf{2 0 1 1}$ & $\mathbf{2 0 1 2}$ & $\mathbf{2 0 1 3}$ \\
\hline ÁLAVA & 0,46 & 0,92 & 0,83 & 0,63 & 0,54 & 0,38 & 0,39 & 0,40 & 0,45 & 0,53 & 0,58 \\
ALBACETE & 0,74 & 0,57 & 0,45 & 0,37 & 0,32 & 0,29 & 0,29 & 0,30 & 0,33 & 0,35 & 0,39 \\
ALICANTE & 0,41 & 0,33 & 0,47 & 0,34 & 0,27 & 0,20 & 0,20 & 0,20 & 0,23 & 0,23 & 0,26 \\
ALMERÍA & 0,72 & 0,66 & 0,71 & 0,34 & 0,23 & 0,19 & 0,21 & 0,22 & 0,27 & 0,30 & 0,36 \\
ASTURIAS & 1,00 & 0,83 & 0,69 & 0,61 & 0,42 & 0,37 & 0,38 & 0,39 & 0,39 & 0,41 & 0,49 \\
ÁVILA & 0,97 & 0,55 & 0,51 & 0,50 & 0,45 & 0,38 & 0,39 & 0,41 & 0,45 & 0,44 & 0,43 \\
BADAJOZ & 0,66 & 0,62 & 0,47 & 0,38 & 0,30 & 0,26 & 0,26 & 0,26 & 0,27 & 0,29 & 0,31 \\
BALEARES & 0,47 & 0,44 & 0,41 & 0,31 & 0,30 & 0,27 & 0,28 & 0,28 & 0,30 & 0,36 & 0,41 \\
BARCELONA & 0,19 & 0,33 & 0,26 & 0,21 & 0,17 & 0,15 & 0,15 & 0,19 & 0,21 & 0,28 & 0,42 \\
BURGOS & 0,49 & 0,45 & 0,41 & 0,37 & 0,33 & 0,29 & 0,29 & 0,31 & 0,31 & 0,39 & 0,42 \\
CÁCERES & 0,50 & 0,53 & 0,47 & 0,40 & 0,38 & 0,37 & 0,38 & 0,38 & 0,38 & 0,40 & 0,43 \\
CÁDIZ & 0,28 & 0,26 & 0,38 & 0,26 & 0,22 & 0,19 & 0,19 & 0,20 & 0,21 & 0,37 & 0,41 \\
CANTABRIA & 1,00 & 0,56 & 0,55 & 0,46 & 0,41 & 0,36 & 0,36 & 0,38 & 0,39 & 0,40 & 0,44 \\
CASTELLÓN & 0,43 & 0,46 & 0,58 & 0,34 & 0,27 & 0,21 & 0,22 & 0,24 & 0,28 & 0,33 & 0,34 \\
CEUTA & & & 0,78 & 0,42 & 0,34 & 0,30 & 0,30 & 0,33 & 0,31 & 0,36 & 0,36
\end{tabular}

El número equivalente de empresas se calcula como el inverso del índice de Herfindahl: $\mathrm{n}^{\circ}=1 / \mathrm{H}$ y refleja el número de empresas del mismo tamaño que participan en el mercado, con un nivel de competencia similar al existente. 


\begin{tabular}{|c|c|c|c|c|c|c|c|c|c|c|c|}
\hline CIUDAD & 0,29 & 0,28 & 0,32 & 0,26 & 0,24 & 0,22 & 0,22 & 0,23 & 0,24 & 0,25 & 0,26 \\
\hline CÓRDOBA & 0,42 & 0,39 & 0,72 & 0,42 & 0,32 & 0,29 & 0,29 & 0,29 & 0,29 & 0,34 & ),39 \\
\hline CUENCA & 0,97 & 0,80 & 0,81 & 0,64 & 0,60 & 0,53 & 0,53 & 0,56 & 0,57 & 0,58 & 0,66 \\
\hline GERONA & 0,30 & 0,25 & 0,25 & 0,22 & 0,20 & 0,19 & 0,18 & 0,34 & 0,32 & 0,39 & 0,61 \\
\hline GRANADA & 0,55 & 0,52 & 0,66 & 0,50 & 0,42 & 0,35 & 0,36 & 0,35 & 0,32 & 0,34 & 0,39 \\
\hline UADALAJARA & 0,47 & 0,43 & 0,42 & 0,35 & 0,27 & 0,22 & 0,23 & 0,25 & 0,28 & 0,37 & 0,38 \\
\hline GUIPÚZCOA & 0,51 & 0,99 & 0,90 & 0,62 & 0,54 & 0,41 & 0,41 & 0,42 & 0,43 & 0,52 & 0,55 \\
\hline HUELVA & 0,47 & 0,47 & 0,44 & 0,30 & 0,24 & 0,28 & 0,27 & 0,27 & 0,29 & 0,52 & 0,52 \\
\hline HUESCA & 0,83 & 0,73 & 0,58 & 0,50 & 0,43 & 0,41 & 0,42 & 0,44 & 0,44 & 0,45 & 0,58 \\
\hline JAÉN & 0,31 & 0,29 & 0,25 & 0,20 & 0,18 & 0,17 & 0,17 & 0,22 & 0,22 & 0,24 & 0,25 \\
\hline LA CORUÑA & 0,95 & 0,64 & 0,54 & 0,44 & 0,36 & 0,32 & 0,32 & 0,47 & 0,44 & 0,43 & 0,49 \\
\hline OJA & 0,50 & 0,46 & 0,41 & 0,35 & 0,30 & 0,27 & 0,27 & 0,28 & 0,31 & 0,35 & 0,38 \\
\hline LAS PALMAS & 0,91 & 0,65 & 0,55 & 0,41 & 0,32 & 0,25 & 0,25 & 0,25 & 0,36 & 0,45 & 0,48 \\
\hline LEÓN & 1,00 & 0,87 & 0,44 & 0,39 & 0,33 & 0,27 & 0,28 & 0,31 & 0,33 & 0,33 & 0,48 \\
\hline LÉRIDA & 0,28 & 0,31 & 0,24 & 0,23 & 0,22 & 0,21 & 0,21 & 0,25 & 0,27 & 0,32 & 0,41 \\
\hline LUGO & 0,93 & 0,70 & 0,49 & 0,45 & 0,45 & 0,41 & 0,42 & 0,60 & 0,58 & 0,59 & 0,61 \\
\hline MADRID & 0,80 & 0,58 & 0,39 & 0,25 & 0,20 & 0,17 & 0,18 & 0,19 & 0,22 & 0,25 & 0,27 \\
\hline MÁL & 0,37 & 0,32 & 0,65 & 0,45 & 0,30 & 0,22 & 0,23 & 0,24 & 0,26 & 0,29 & 0,36 \\
\hline MELILLA & & & 1,00 & 0,44 & 0,43 & 0,34 & 0,34 & 0,34 & 0,34 & 0,43 & 0,43 \\
\hline MURCIA & 0,50 & 0,44 & 0,43 & 0,32 & 0,28 & 0,23 & 0,24 & 0,24 & 0,25 & 0,34 & 0,42 \\
\hline NAVARRA & 0,56 & 0,47 & 0,41 & 0,58 & 0,49 & 0,42 & 0,41 & 0,43 & 0,44 & 0,65 & 0,74 \\
\hline ORENSE & 0,71 & 0,45 & 0,41 & 0,43 & 0,41 & 0,39 & 0,39 & 0,75 & 0,68 & 0,68 & 0,66 \\
\hline PALENCIA & 0,55 & 0,40 & 0,34 & 0,30 & 0,27 & 0,24 & 0,24 & 0,35 & 0,37 & 0,39 & 0,48 \\
\hline PONTEVEDRA & 0,45 & 0,35 & 0,31 & 0,47 & 0,37 & 0,33 & 0,32 & 0,51 & 0,47 & 0,46 & 0,49 \\
\hline SALAMANCA & 1,00 & 0,88 & 0,74 & 0,55 & 0,45 & 0,33 & 0,34 & 0,42 & 0,45 & 0,46 & 0,50 \\
\hline SEGOVIA & 1,00 & 0,91 & 0,80 & 0,68 & 0,66 & 0,50 & 0,52 & 0,53 & 0,64 & 0,65 & 0,65 \\
\hline SEVILLA & 0,39 & 0,36 & 0,32 & 0,22 & 0,18 & 0,25 & 0,24 & 0,24 & 0,25 & 0,47 & 0,47 \\
\hline SORIA & 1,00 & 0,90 & 0,79 & 0,70 & 0,64 & 0,50 & 0,51 & 0,53 & 0,52 & 0,56 & 0,59 \\
\hline TARRAGONA & 0,28 & 0,26 & 0,29 & 0,28 & 0,23 & 0,21 & 0,21 & 0,27 & 0,28 & 0,33 & 0,46 \\
\hline TENERIFE & 1,00 & 0,74 & 0,63 & 0,51 & 0,45 & 0,39 & 0,39 & 0,41 & 0,43 & 0,76 & 0,84 \\
\hline TERUEL & 0,86 & 0,83 & 0,68 & 0,64 & 0,59 & 0,57 & 0,57 & 0,58 & 0,58 & 0,60 & 0,68 \\
\hline TOLEDO & 0,83 & 0,57 & 0,54 & 0,47 & 0,39 & 0,35 & 0,35 & 0,36 & 0,37 & 0,39 & 0,44 \\
\hline VALENCIA & 0,53 & 0,40 & 0,41 & 0,24 & 0,22 & 0,18 & 0,19 & 0,19 & 0,23 & 0,30 & 0,29 \\
\hline VALLADOLID & 0,37 & 0,57 & 0,42 & 0,34 & 0,25 & 0,21 & 0,22 & 0,33 & 0,35 & 0,31 & 0,42 \\
\hline VIZCAYA & 0,50 & 0,94 & 0,81 & 0,53 & 0,46 & 0,35 & 0,35 & 0,35 & 0,38 & 0,43 & 0,48 \\
\hline ZAMORA & 0,68 & 0,57 & 0,55 & 0,51 & 0,42 & 0,38 & 0,38 & 0,56 & 0,56 & 0,57 & 0,64 \\
\hline ZARAGOZA & 0,54 & 0,47 & 0,41 & 0,32 & 0,26 & 0,23 & 0,24 & 0,25 & 0,26 & 0,29 & 0,35 \\
\hline
\end{tabular}

Fuente: Elaboración propia

\section{Patron de comportamiento de las Cajas de Ahorros}

En este epígrafe se analiza la evolución que han tenido las cajas de ahorros como entidad a lo largo del tiempo. Como se ha comentado anteriormente, tras la crisis económica y financiera del 2008 se produce una reestructuración que afectó considerablemente a las cajas de ahorros y que provocó que en 2013 quedaran 
solamente 15 entidades o grupos de entidades ${ }^{6}$. En ese momento se dio comienzo a una serie de fusiones tanto convencionales, que son aquellas que se llevan a cabo por cajas de ahorros pertenecientes a la misma región en las que las alianzas dan lugar a una única caja con personalidad jurídica independiente de las cajas que se han unido, como SIP o "fusiones frías" que han sido la alternativa más utilizada para fusionarse aquellas entidades que no pertenecen a la misma región.

Así, teniendo en cuenta las fusiones y agrupaciones que se han dado entre las diferentes cajas de ahorros hemos elaborado el cuadro 4, en donde se recoge el número de oficinas para cada una de las 15 entidades existentes en 2013, teniendo en cuenta los datos históricos de las setenta y seis entidades que existían al comienzo de nuestro periodo de estudio y que se han ido incorporando durante los 28 años de estudio. De esta manera podemos ver cómo han evolucionado las entidades a lo largo de los años si hubieran tenido siempre la estructura del último año. Cabe destacar que dos entidades se integraron en bancos independientes al sector de las cajas de ahorros, como fue el caso del Banco CAM que se integró en el Banco Sabadell en 2011 y el Unnim Banc que se integró en el BBVA en 2012.

6 En la actualidad existen en nuestro país once entidades. 
Gráfico. 1. Proceso de reestructuración del sector cajas de ahorros, 2009-2013

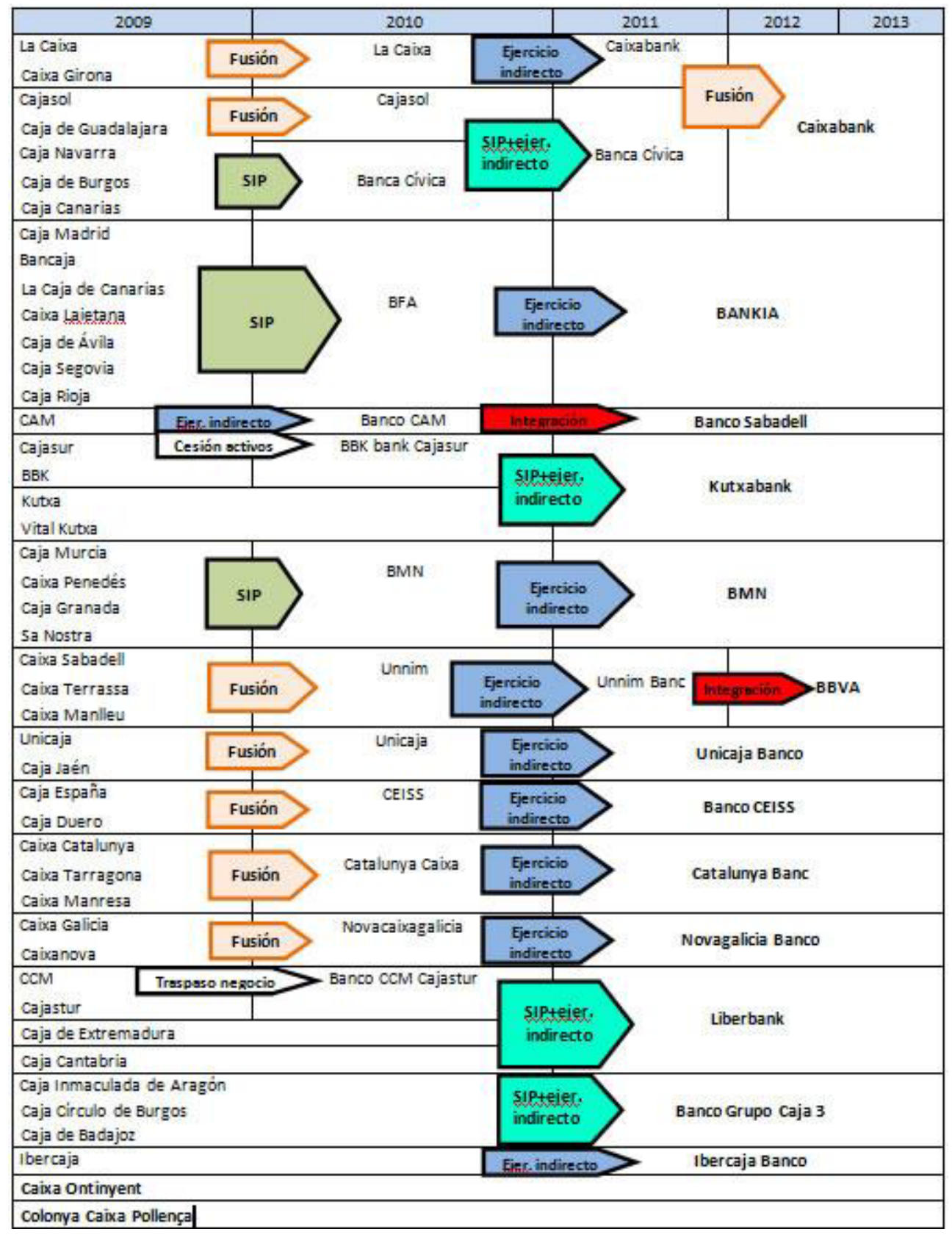

Fuente: Elaboración propia con datos de la CECA 
Cuadro. 4. Número de oficinas por entidad, 1986-2013

\begin{tabular}{|c|c|c|c|c|c|c|c|c|c|c|c|}
\hline & 1986 & 990 & 95 & 000 & 2005 & 008 & 2009 & 2010 & 2011 & 2012 & 01 \\
\hline & & +0) & & & & 470 & & & & & 0.07 \\
\hline & 24 & 17 & 70 & & 1 & 56 & 9 & & & & (0) \\
\hline & 25 & 928 & 19 & & & 1.672 & 1.605 & & & & 1.009 \\
\hline NCG & 6 & 80 & 7 & & & 47 & & & & 26 & 580 \\
\hline & 804 & 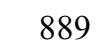 & 674 & 8 & 9 & .377 & $1 .$. & & & 06 & 1.061 \\
\hline & 752 & 1.250 & 986 & 1.279 & 2.096 & .341 & & & & 1.857 & 2.418 \\
\hline m & 5 & 911 & 1.071 & 1.409 & 1.683 & 1.821 & 1.741 & & & & 818 \\
\hline & 746 & 769 & 723 & 766 & 896 & 970 & & 31 & & 01 & 753 \\
\hline $\begin{array}{r}\text { Banco } \\
\text { grupo }\end{array}$ & 35 & 30 & 521 & 652 & 08 & 1.085 & 1085 & 76 & 2 & 47 & 1.016 \\
\hline Caj & 356 & 389 & 475 & 536 & 604 & 625 & 616 & 595 & 575 & 575 & 389 \\
\hline & & 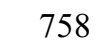 & 837 & 1.088 & 9 & 1.148 & & & & 79 & 722 \\
\hline & . & 87 & 878 & 984 & 1.140 & 1.366 & 1.33 & & & & 1.136 \\
\hline BBVA & 2.726 & 2.730 & 2.568 & 4.341 & 4.136 & 4.091 & 3.816 & 3.717 & 3.608 & 3.028 & 3.247 \\
\hline yent & 19 & 27 & 28 & 33 & 42 & 47 & 47 & 47 & 47 & 47 & 47 \\
\hline Caixa Pollença & 5 & 8 & 11 & 13 & 17 & 21 & 21 & 21 & 21 & 20 & 20 \\
\hline
\end{tabular}

Fuente: Elaboración propia

Ahora vamos a ir analizando cada fusión para ver sus rasgos más relevantes. Todas las entidades son el resultado de un proceso de reestructuración tras la sobredimensión en el número de oficinas, en el año 2008. A partir de ese momento, la reestructuración del sector era obligada debido a las limitaciones estructurales asociadas a su naturaleza jurídica.

El Real Decreto-Ley 9/2009, del 26 de junio, conocido como "Ley del FROB", impulsó un intenso proceso de reestructuración del sector, un proceso muy laborioso de integraciones, fusiones y ajustes normativos. También fue relevante la reforma del marco jurídico de las cajas de ahorros producida por el Real DecretoLey 11/2010, de 9 de julio, que dio la posibilidad de que las cajas de ahorros pudieran desarrollar su actividad a través de un banco (ejercicio indirecto de actividad) con una participación de más del $50 \%$ de sus derechos de voto y que de esta manera les permitiera acceder a los mercados de capitales para reforzar sus recursos propios de primera calidad e introducir mejoras en su gobernanza. Por último, el Real Decreto-Ley 2/2011, el 18 de febrero que reforzó el sistema financiero español y trato de conseguir el saneamiento de las cajas.

Como resultado de todo esto, en 2013, estas eran las entidades resultantes:

CaixaBank: resultado de la absorción de fusión de Banca Cívica en 2012 (Sistema Institucional de Protección formado por Caja Navarra, Cajasol, Caja 
Canarias y Caja Burgos y la Caja de Ahorros y Pensiones de Barcelona ("La Caixa"), por tanto, es la que más cajas ha integrado por el proceso de reestructuración. Destaca que desde su inicio ya estaba situada en la mayoría de las provincias de España por lo que ha tenido una influencia nacional (en 1990 ya estaba situada en 48 provincias y desde 1996 en las 52) y gracias a la fusión con Banca Cívica en 2012 supera las 6.000 oficinas en el territorio nacional.

Bankia: Surge tras la fusión de los negocios bancarios que se dan en 2011 de Caja Madrid, Bancaja, Caja Canarias, Caja Ávila, Caixa Laietana, Caja Segovia y Caja Rioja. En 1986 estaba únicamente situado en 14 provincias pero pronto comienza su proceso de expansión y es en 1994 cuando ya se encuentra en 51 provincias (en todas salvo en Melilla). En 2013, se redujeron unas 1.000 oficinas, sin afectar esto a su localización en las provincias.

CatalunyaBank: surge de la fusión de Caixa d'Estalvis de Catalunya, Caja Manresa y Caja Tarragona. El FROB aprobó la ayuda que solicitó CatalunyaCaixa, la cual estaba condicionada a la conversión en banco del negocio financiero de CatalunyaCaixa, lo que se hizo mediante la entidad CatalunyaBank, y a la recapitalización mediante la entrada de inversión privada en el capital del banco.

En 1986 en tan solo 6 provincias (Barcelona, Madrid, Valencia, Lleida, Tarragona y Girona) y poco a poco se fue expandiendo hasta llegar a las 50 provincias a partir de 2001. En 2013 mantiene esta cifra y destaca porque el proceso de reestructuración no le afectó tan gravemente como a otras entidades.

NCG banco: surge de la fusión de Caixa Galicia y Caixanova (la cual anteriormente se había fusionado con la Caja de Orense y Pontevedra). En el año 2011 estaba situado en 50 provincias con 1.029 oficinas y en el año 2013 en 25 provincias con 580 entidades. Es decir, no solo se reduce el número de oficinas casi a la mitad, sino también el número de entidades en un $50 \%$ en apenas dos años.

Kutxabank: se crea como resultado de la fusión fría del negocio bancario de las cajas BBK (formada por la fusión de la Caja Bilbao y Vizcaína), Kutxa (formada por la fusión de las cajas Guipúzcoa y San Sebastián) y Caja Vital (como consecuencia de la unión de las cajas Vitoria y Álava), que traspasaron su negocio financiero a dicha entidad. En Andalucía y Extremadura opera a través de su filial CajaSur Banco. En el año 2013 contaba con 1.061 oficinas (de las cuales 387 pertenecen a su filial CajaSur Banco) y se encontraba en 30 provincias.

Banco Sabadell: fue fundado en 1881. En el año 2011 se le adjudicó en una subasta el Banco CAM (fruto de las fusiones de las cajas Torrent y Pr. AlicanteValencia), que estaba nacionalizado por el Banco de España por sus problemas financieros. Está presente en 51 provincias y es el cuarto mayor grupo financiero español y tiene más ofícinas (casi 600 más) en el año 2013 que en el 2008.

Banco Mare Nostrum: es un banco español que nació en 2010 como Sistema Institucional de Protección (SIP) del negocio financiero de cuatro cajas de ahorros: Caja Murcia, Caixa Penedès, Caja Granada y Sa Nostra. Opera en comunidades autónomas del arco del mediterráneo así como la Comunidad de Madrid, estando presente en 24 provincias en 2011 y 2012. En 2013 se reducen a 19, con una reducción en el número de oficinas de más de 500.

Unicaja Banco: es una entidad financiera que se crea en diciembre de 2011 con el traspaso de la actividad de Unicaja, conforme a lo dispuesto en el artículo 5 del Real Decreto-Ley 11/2010. Proviene de la fusión de Monte de Piedad y Caja de 
Ahorros de Ronda, Cádiz, Almería, Málaga, Antequera y Jaén. Es el sexto grupo bancario privado español, y se encuentra en el año 2013 en 20 provincias, teniendo una importante presencia territorial principalmente en las comunidades autónomas de Andalucía y Castilla y León.

Ibercaja Banco: Se creó en 2011 por la Caja de Ahorros y Monte de Piedad de Zaragoza, Aragón y Rioja (Ibercaja) para desarrollar su actividad financiera. En el año 1986 se encontraba en tan solo 8 provincias y en el 2013 en 50, destacando el salto que dio en el año 2001 situándose en 11 nuevas provincias respecto al año anterior. En lo que respecta al número de oficinas también mostró un crecimiento relevante pasando de 355 oficinas en 1986 y superando las 1.000 desde el 2005 en adelante, obteniendo su máximo en los años 2008 y 2009 (1.085 oficinas).

Banco Grupo Cajatres: Se creó en 2011 a partir de la integración de la actividad financiera de las cajas: Caja Inmaculada (CAI), Caja Círculo de Burgos y Caja de Badajoz mediante un Sistema Institucional de Protección (SIP). Con la integración de las tres cajas de ahorros pasó de estar situada en 12 provincias (en 2010) a estar en 33 provincias en 2011. En 2012 mantuvo esta cifra, pero en el siguiente año se redujo gravemente a más de la mitad, pasando a estar finalmente en 16.

Banco CEISS: oficialmente "Banco de Caja España de Inversiones, Salamanca y Soria”, es la entidad bancaria creada por Caja España-Duero en 2011 a través de la que desarrolla su actividad financiera (Caja España estaba constituida por la caja de León, Zamora, Popular de Valladolid, Provincial de Valladolid y Palencia y Caja Duero por las cajas de Salamanca y Soria). Opera principalmente en Castilla y León, Cáceres y Madrid, aunque también tiene presencia en otras zonas de la geografía española, estando en un total de 39 provincias en 2013.

Liberbank: banco constituido en 2011 mediante un SIP por Cajastur, Caja de Extremadura y Caja Cantabria, al que aportaron los activos y pasivos del negocio bancario. En Castilla-La Mancha opera a través de su filial CCM Banco. En 2012 se aprobó su fusión con Ibercaja Banco y Caja 3, de la cual nacería la séptima entidad financiera española, pero dicha fusión se canceló como resultado del test de estrés de Oliver Wyman y las exigencias de capital que Liberbank mostraba en los escenarios de dicho documento. La crisis no ha hecho que se reduzca gravemente su número de oficinas y provincias en las que está situada.

BBVA: aprovechó la reestructuración del sistema financiero de España para crecer especialmente en Cataluña, una de las regiones donde menos cuota de mercado tenía. Por ello en 2012 adquiere a Unnim Banc, un grupo financiero nacionalizado por el Gobierno de España, que agrupaba a las cajas de ahorros Caixa Sabadell, Caixa Terrassa y Caixa Manlleu. Durante todo el periodo de estudio 1986-2013 se encuentra situado en las 52 provincias y es el segundo grupo financiero español. Por su naturaleza de banco se ve también menos afectado por la crisis, como el caso del Banco Sabadell, incrementándose en el año 2013 más de 200 oficinas respecto al año anterior, siendo las dos únicas entidades, de las quince, que aumentaron oficinas en este año.

Caixa Ontinyent: es una caja de ahorros española fundada en 1884 en la localidad Valenciana de Onteniente. Hasta el año 2000 solo se encontraba situada en Valencia y a partir de este año se encuentra también en Alicante. Durante la crisis no se ha reducido su número de oficinas en ningún año, siendo este 47. 
Colonya Caixa Pollença: es una caja de ahorros española fundada en 1880 en Pollensa (Islas Baleares). Es, junto a la Caixa Ontinyent, las dos cajas de ahorros españolas que existen en la actualidad, ya que no se vieron afectadas por la reestructuración del sector ocurrida tras la crisis financiera del 2008. Durante todo el periodo 1986-2013 solo se encuentra situada en la provincia de Baleares, en la cual antes de la crisis tenía 21 oficinas y posteriormente se han reducido a 20.

Como conclusión hemos podido observar algunos patrones ${ }^{7}$ que se repiten en el comportamiento de las entidades:

1. Algunas entidades reducen bastante en el último año (2013) su localización en las provincias, como es el caso de NCG banco, Banco Caja 3 y Banco CEISS (los dos primeros a la mitad y el tercero dejo de estar en 10 provincias).

2. Entidades como BBVA y Caixabank destacan porque durante todo el periodo han estado presentes en casi todas las provincias.

3. Otras como Bankia, CatalunyaBank, Banco Sabadell e Ibercaja destacan porque en los primeros años se situaban en pocas provincias pero han tenido grandes procesos de expansión acabando en casi todo el territorio nacional.

4. Algunas como Ontinyent y Pollença se han mantenido similares durante todo el periodo, sin grandes cambios. ${ }^{8}$

5. Por último, algunas entidades destacan por una pequeña expansión a lo largo de los años y por reducirse su localización en provincias únicamente en el año 2013 (aunque son reducciones poco significativas en comparación con las del grupo 1). Ejemplo de estas pueden ser Kutxabank, BMN, Liberbank.

\section{Conclusiones}

Las cajas de ahorros han experimentado una gran reestructuración que se ha traducidom entre otras cosas, a una reducción del número de entidades, de oficinas y del número de provincias en la que tienen presencia.

Durante el primer periodo analizado (1986-2008), se produjo una gran expansión, que tuvo como resultado la apertura de un elevado número de oficinas, al tiempo que las entidades comenzaron un importante proceso de fusiones, lo que provocó que se pasara de 76 a 45 cajas de ahorros.

En 2013, quedaron solamente 15 entidades, de las que únicamente mantienen su estructura y modelo tradicional de cajas de ahorros la de Ontinyent y la de Pollença. Estas dos entidades, representan sólo un $0,1 \%$ del negocio bancario del total de las cajas pero han sabido sobrevivir (Gutierrez, Fernández Y Palomo, 2016)

7 Para mayor información sobre la materia véase el artículo Delgado, Saurina y Towwnsend (2008) "Estrategias de expansión de las entidades de depósito españolas. Una primera aproximación descriptiva" en Estabilidad Financiera, No 15, Banco de España, p. 107 en adelante. También Fuentelsaz y Gómez (2001).

8 Solamente estas dos entidades han conseguido conservarse, sin necesidad de haberse integrado con otras entidades ni de convertirse en bancos (representan tan sólo un $0.1 \%$ del negocio bancario). Según Gutierrez, Fernández y Palomo (2016) se puede deber a ser las más pequeñas del sector, pero principalmente por ser las únicas que cumplen con los criterios de banca minorista y de limitación geográfica en que se ha basado tradicionalmente el modelo de negocio de cajas de ahorros. 
En el presente trabajo también se ha analizado los cambios que se han producido en la estructura de las cincuenta y dos provincias, observando el número de oficinas de cajas de ahorros y el nivel de concentración alcanzado en cada uno de ellos (la competencia), observándose que en la mayor parte de las provincias se reducía la concentración, lo que confirmaba un aumento en la rivalidad entre las cajas de ahorros, lógico por el proceso de liberalización que sufrieron a finales de los noventa.

En el análisis por entidad profundizamos caso a caso las 15 entidades que quedaban en 2013 (CaixaBank, Bankia, Catalunya Banc, NCG Banco, Kutxabank, Banco Sabadell, Banco Mare Nostrum, Unicaja Banco, Ibercaja Banco, Banco Grupo Cajatres, Banco CEISS, Liberbank, BBVA, Caja de Ontinyent y Caixa Pollença $)^{9}$ identificando algunos patrones de comportamiento similares entre ellas. Las entidades que más se han visto afectadas en sus resultados han sido Caixabank, Kutxabank y Ontinyent (Gutierrez, Fernández Y Palomo, 2016).

A pesar de todo esto, con la reestructuración que se llevó a cabo tenemos un sector bancario más solvente y saneado, ya que se han corregido gran parte de los desequilibrios que tenía en el pasado. Sin embargo, aun tiene que enfrentarse a retos importantes, sobre todo los derivados del incierto escenario macroeconómico. Vemos que la reestructuración en España ha sido muy considerable, con una intensidad prácticamente inédita en Europa. Los resultados de algunos estudios recientes sugieren la lenta pero continua recuperación de este sector en unos años.

\section{Referencias bibliográficas}

Anuarios estadísticos de la AEB. Disponibles en: http://www.aebanca.es/Publicaciones/AnuarioEstad\%C3\%ADstico/index.htm

Anuarios estadísticos de la CECA, desde 1986 a 2013. Disponibles en: http://www.ceca.es/que-hacemos/analisis-economico-y-regulatorio/estados-financieros/

Berges, A. (2003) Bancos y cajas: estrategias divergentes. Análisis financiero internacional, $\mathrm{n}^{\circ} 1112$, pp. 5-20.

Bernad, C., Fuentelsaz, L., y Gómez, J. (2005) Consolidación, expansión y localización de las oficinas bancarias en España, 1995-2002. Perspectivas del sistema financiero, $\mathrm{n}^{\circ}$ 83, pp. 5-65.

Calvo, A.; Parejo, J. A.; Rodríguez, L. y Cuervo, A. (2010) Manual del sistema financiero español, $22^{\mathrm{a}}$ edición, Ariel, Barcelona.

Delgado, J., Saurina, J. y Townsend, R. (2008) Estrategias de expansión de las entidades de depósito españolas. Una primera aproximación descriptiva. Estabilidad Financiera, $\mathrm{n}^{\circ}$ 15, Banco de España, p. 107 en adelante.

Fuentelsaz, L. y Gómez, J. (2001) Liberalización y estrategia competitiva: la expansión de las cajas de ahorros. Cuadernos de Información Económica, $\mathrm{n}^{\circ}$ 164, pp.74-85.

Fuentelsaz, L., Gómez, J. y Polo, Y. (2002) La expansión de la red de oficinas de las cajas de ahorros: una perspectiva estratégica. Perspectivas del Sistema Financiero, $\mathrm{n}^{\mathrm{o}}$ 80, pp. $57-71$.

9 Dentro de Kutxabank está integrado CajaSur Banco y dentro de Liberbank está integrado Banco Castilla-La Mancha. 
González, J y González, J.P. (2012) Las cajas de ahorros en el Sistema financiero español. Tribuna de economía ICE, $\mathrm{n}^{\mathrm{o}}$ 867, pp. 141-156.

Gutiérrez, M., Fernández Y., y Palomo, R. (2016) De la transformación a la bancarización de las cajas de ahorros españolas: Un análisis de los resultados perseguidos. REVESCO. Revista de Estudios Cooperativos, $\mathrm{n}^{\mathrm{o}}$ 122, pp. 86-109. DOI: 10.5209/rev_REVE.2016.v122.52022.

Gutiérrez, M., Palomo, R. y Fernández, G. (2013) Las cajas de ahorros españolas: ¿una pretendida reordenación bajo criterios de racionalidad económica y social?. Cuadernos de Economía y Dirección de la Empresa, vol. 16, no 4, pp. 250-268

Gutiérrez, M., Palomo, R. y Romero, M. (2012) La expansión territorial como factor motivador de la reestructuración del sistema financiero español: El caso de las cajas de ahorros y las cooperativas de crédito. REVESCO. Revista de Estudios Cooperativos, $\mathrm{n}^{\circ}$ 107, pp. 7-34. DOI: 10.5209/rev_REVE.2012.v107.38746.

Ibarrondo, P. y Sánchez, J. (2005) Tendencias estratégicas de las cajas de ahorro y las cajas rurales españolas. Investigaciones europeas de dirección y economía de la empresa, vol. $11, \mathrm{n}^{\mathrm{o}} 1$, pp. 119-142.

Illueca, M., Pastor, J.M., y Tortos, E. (2005) El efecto de la expansión geográfica sobre la productividad de las cajas de ahorro españolas. Perspectivas del sistema financiero, $\mathrm{n}^{\circ}$ 83, pp 37-54.

Maudos, J. (2016) La concentración regional del mercado bancario. Cuadernos de información económica, $\mathrm{n}^{\circ} 251$, pp. 49-62

Palomo, R., y González, M. (2004) Un contraste de la divergencia en el modelo de negocio de las entidades financieras de economía social: cajas de ahorro y cooperativas de crédito. REVESCO. Revista de estudios Cooperativos, $\mathrm{n}^{\circ}$ 83, pp. 85-114.

Palomo, R., y Gutiérrez, M. (2012) El desajuste del crédito en el sistema bancario y la acción de la economía social: el camino de la reestructuración. REVESCO. Revista de estudios Cooperativos, $\mathrm{n}^{\mathrm{o}}$ 109, pp. 138-169. DOI: 10.5209/ rev_REVE.2012.v109.40653.

Palomo, R., y Sanchis, J.R. (2008) Un análisis del crédito cooperativo en España: situación actual, expansión territorial y proyección estratégica. Estudios de Economía Aplicada, vol. $1, \mathrm{n}^{\mathrm{o}} 26$, pp. 89-132. 
Anexo. 1. Evolución del número de oficinas de las cajas de ahorro y número de provincias en las que están presentes 1986-2013

\begin{tabular}{|c|c|c|c|c|c|c|c|c|c|c|c|c|c|c|c|c|c|c|c|c|c|c|c|c|c|c|c|c|c|}
\hline & & 1986 & 1987 & 1988 & 1989 & 1990 & 1991 & 1992 & 1993 & 1994 & 1995 & 1996 & 1997 & 1998 & 1999 & 2000 & 2001 & 2002 & 2003 & 2004 & 2005 & 2006 & 2007 & 2008 & 2009 & 2010 & 2011 & 2012 & 2013 \\
\hline \multirow{2}{*}{ BANKIA } & oficinas & 699 & 774 & 819 & 832 & 918 & 1064 & 1129 & 1192 & 1263 & 1301 & 1366 & 1452 & 1545 & 1585 & 1762 & 1839 & 1871 & 1875 & 1879 & 1902 & 1945 & 1977 & 2092 & 2109 & 2089 & 3168 & 3037 & 2092 \\
\hline & no provincias & 14 & 20 & 21 & 21 & 41 & 47 & 47 & 47 & 51 & 51 & 51 & 51 & 51 & 51 & 51 & 51 & 51 & 51 & 51 & 51 & 51 & 51 & 51 & 51 & 51 & 51 & 51 & 51 \\
\hline \multirow{2}{*}{ CAJA CARLET } & oficinas & 8 & 8 & 11 & 11 & 12 & 12 & 12 & 12 & 12 & 12 & 13 & 15 & 15 & 16 & 16 & - & - & - & - & - & - & - & - & - & - & - & - & - \\
\hline & no provincias & 1 & 1 & 1 & 1 & 1 & 1 & 1 & 1 & 1 & 1 & 1 & 1 & 1 & 1 & 1 & - & - & - & - & - & - & - & - & - & - & - & - & - \\
\hline CAJA CASTELLÓN & no provincias & 2 & 2 & 2 & 2 & 2 & - & - & - & - & - & - & - & - & - & - & - & - & - & - & - & - & - & - & - & - & - & - & - \\
\hline \multirow{2}{*}{ CAAA RIOJA } & oficinas & 94 & 97 & 98 & 109 & 113 & 105 & 105 & 102 & 102 & 105 & 102 & 103 & 103 & 105 & 106 & 109 & 110 & 113 & 116 & 117 & 117 & 120 & 122 & 118 & 118 & - & - & - \\
\hline & no provincias & 1 & 1 & 1 & 1 & 4 & 5 & 4 & 5 & 4 & 4 & 4 & 4 & 4 & 5 & 5 & 5 & 6 & 6 & 7 & 8 & 8 & 8 & 8 & 8 & 8 & - & - & - \\
\hline CAJA LAIETANA & oficinas & 83 & 86 & 88 & 93 & 97 & 101 & 105 & 111 & 116 & 124 & 134 & 144 & 156 & 169 & 184 & 200 & 217 & 233 & 250 & 258 & 267 & 275 & 277 & 264 & 256 & - & - & - \\
\hline \multirow{2}{*}{ CANARIAS INS } & oficinas & 106 & 109 & 114 & 116 & 116 & 111 & 111 & 114 & 113 & 114 & 114 & 116 & 124 & 128 & 129 & 134 & 142 & 153 & 158 & 160 & 170 & 180 & 184 & 184 & 165 & - & - & - \\
\hline & no provincias & 1 & 2 & 3 & 3 & 3 & 3 & 3 & 3 & 3 & 3 & 3 & 3 & 3 & 3 & 3 & 3 & 3 & 3 & 3 & 3 & 3 & 3 & 3 & 3 & 3 & - & - & - \\
\hline \multirow{2}{*}{ CAJA SAGUNTO } & oficinas & 36 & 38 & 41 & 41 & 41 & 46 & 46 & - & - & - & - & - & - & - & - & - & - & - & - & - & - & - & - & - & - & - & - & - \\
\hline & no provincias & 1 & 1 & 2 & 2 & 2 & 2 & 2 & - & - & - & - & - & - & - & - & - & - & - & - & - & - & - & - & - & - & - & - & - \\
\hline \multirow{2}{*}{ CAJA SEGORBE } & oficinas & 23 & 23 & 24 & - & - & - & - & - & - & - & - & - & - & - & - & - & - & - & - & - & - & - & - & - & - & - & - & - \\
\hline & no provincias & 1 & 1 & 1 & - & - & - & - & - & - & - & - & - & - & - & - & - & - & - & - & - & - & - & - & - & - & - & - & - \\
\hline \multirow{2}{*}{ CAJA SEGOVIA } & oficinas & 58 & 59 & 61 & 61 & 61 & 63 & 63 & 63 & 63 & 63 & 63 & 73 & 78 & 90 & 92 & 95 & 97 & 99 & 102 & 103 & 108 & 112 & 116 & 117 & 114 & - & - & - \\
\hline & no provincias & 2 & 2 & 2 & 2 & 2 & 3 & 3 & 3 & 3 & 3 & 3 & 3 & 3 & 3 & 3 & 3 & 3 & 3 & 3 & 3 & 3 & 4 & 5 & 5 & 4 & - & - & - \\
\hline \multirow{2}{*}{ BANCAJA } & oficinas & 461 & 469 & 488 & 526 & 479 & 628 & 629 & 655 & 655 & 659 & 656 & 673 & 673 & 704 & 758 & 767 & 787 & 825 & 937 & 1031 & 1054 & 1111 & 1137 & 1139 & 1128 & - & - & - \\
\hline & no provincias & 7 & 6 & 7 & 7 & 7 & 8 & 8 & 8 & 8 & 10 & 10 & 14 & 14 & 15 & 19 & 19 & 21 & 30 & 45 & 50 & 50 & 50 & 50 & 50 & 50 & - & - & - \\
\hline CAJA ÁVILA & no provincias & 2 & 2 & 2 & 2 & 2 & 2 & 2 & 2 & 2 & 2 & 2 & 2 & 2 & 2 & 2 & 2 & 2 & 3 & 3 & 3 & 4 & 4 & 6 & 6 & 6 & - & - & - \\
\hline
\end{tabular}




\begin{tabular}{|c|c|c|c|c|c|c|c|c|c|c|c|c|c|c|c|c|c|c|c|c|c|c|c|c|c|c|c|c|c|}
\hline & & 1986 & 1987 & 1988 & 1989 & 1990 & 1991 & 1992 & 1993 & 1994 & 1995 & 1996 & 1997 & 1998 & 1999 & 2000 & 2001 & 2002 & 2003 & 2004 & 2005 & 2006 & 2007 & 2008 & 2009 & 2010 & 2011 & 2012 & 2013 \\
\hline \multirow{2}{*}{$\begin{array}{c}\text { NCG } \\
\text { banco }\end{array}$} & oficinas & 615 & 639 & 702 & 753 & 805 & 829 & 885 & 904 & 924 & 937 & 1013 & 1015 & 1039 & 1041 & 1100 & 1051 & 1090 & 1126 & 1155 & 1203 & 1288 & 1405 & 1447 & 1281 & 1344 & 1029 & 826 & 580 \\
\hline & $\mathbf{n}^{\circ}$ provincia & 6 & 6 & 6 & 5 & 7 & 8 & 10 & 10 & 12 & 12 & 24 & 24 & 25 & 26 & 37 & 40 & 40 & 41 & 44 & 50 & 50 & 50 & 50 & 50 & 50 & 50 & 48 & 25 \\
\hline \multirow{2}{*}{ Caixanova } & oficinas & 138 & 143 & 154 & 173 & 189 & 193 & 196 & 197 & 198 & 198 & 200 & 201 & 209 & 337 & 449 & 374 & 396 & 425 & 434 & 459 & 491 & 529 & 573 & 470 & - & - & - & - \\
\hline & $\mathrm{n}^{\circ}$ provincias & 3 & 4 & 5 & 5 & 5 & 5 & 5 & 5 & 5 & 5 & 5 & 5 & 5 & 5 & 6 & 6 & 9 & 17 & 18 & 23 & 34 & 40 & 42 & 42 & - & - & - & - \\
\hline \multirow{2}{*}{ Orense } & oficinas & 103 & 105 & 105 & 105 & 110 & 112 & 114 & 117 & 121 & 127 & 130 & 129 & 130 & - & - & - & - & - & - & - & - & - & - & - & - & - & - & - \\
\hline & $\mathrm{n}^{\circ}$ provincias & 5 & 5 & 5 & 5 & 5 & 5 & 5 & 5 & 5 & 5 & 5 & 5 & 5 & - & - & - & - & - & - & - & - & - & - & - & - & - & - & - \\
\hline \multirow{2}{*}{ Pontevedra } & oficinas & 71 & 73 & 73 & 77 & 89 & 101 & 101 & 106 & 106 & 109 & 116 & 116 & 120 & 122 & - & - & - & - & - & - & - & - & - & - & - & - & - & - \\
\hline & $n^{\circ}$ provincias & 3 & 3 & 3 & 3 & 3 & 5 & 5 & 5 & 5 & 5 & 6 & 6 & 6 & 6 & - & - & - & - & - & - & - & - & - & - & - & - & - & - \\
\hline
\end{tabular}

\begin{tabular}{|c|c|c|c|c|c|c|c|c|c|c|c|c|c|c|c|c|c|c|c|c|c|c|c|c|c|c|c|c|c|}
\hline & & 1986 & 1987 & 1988 & 1989 & 1990 & 1991 & 1992 & 1993 & 1994 & 1995 & 1996 & 1997 & 1998 & 1999 & 2000 & 2001 & 2002 & 2003 & 2004 & 2005 & 2006 & 2007 & 2008 & 2009 & 2010 & 2011 & 2012 & 2013 \\
\hline \multirow{2}{*}{ Kutxabank } & oficinas & 864 & 870 & 881 & 882 & 889 & 811 & 799 & 791 & 791 & 674 & 683 & 712 & 692 & 739 & 788 & 838 & 866 & 889 & 932 & 999 & 1075 & 1357 & 1377 & 1357 & 1344 & 1253 & 1206 & 1061 \\
\hline & \begin{tabular}{|l|}
$\mathbf{n}^{\circ}$ \\
provincias
\end{tabular} & - & - & - & - & - & - & - & - & - & - & - & - & - & - & - & - & - & - & - & - & - & - & - & - & - & - & 36 & 30 \\
\hline \multirow{2}{*}{ BBK } & oficinas & - & - & - & - & 315 & 281 & 273 & 266 & 277 & 282 & 280 & 282 & 280 & 285 & 305 & 317 & 325 & 330 & 333 & 354 & 385 & 428 & 430 & 412 & 407 & 395 & - & - \\
\hline & $\mathrm{n}^{0}$ provincias & - & - & - & - & 6 & 7 & 8 & 8 & 8 & 8 & 8 & 8 & 8 & 8 & 10 & 10 & 12 & 12 & 13 & 14 & 18 & 22 & 22 & 23 & 23 & 23 & - & - \\
\hline \multirow{2}{*}{ Bilbao } & oficinas & 148 & 148 & 152 & 153 & - & - & - & - & - & - & - & - & - & - & - & - & - & - & - & - & - & - & - & - & - & - & - & - \\
\hline & $\mathrm{n}^{\circ}$ provincias & 6 & 6 & 6 & 6 & - & - & - & - & - & - & - & - & - & - & - & - & - & - & - & - & - & - & - & - & - & - & - & - \\
\hline \multirow[t]{2}{*}{ Vizcaína } & oficinas & 159 & 159 & 159 & 159 & - & - & - & - & - & - & - & - & - & - & - & - & - & - & - & - & - & - & - & - & - & - & - & - \\
\hline & $\mathrm{n}^{\circ}$ provincias & 4 & 4 & 4 & 4 & - & - & - & - & - & - & - & - & - & - & - & - & - & - & - & - & - & - & - & - & - & - & - & - \\
\hline \multirow{2}{*}{ VITAL } & oficinas & - & - & - & - & 153 & 125 & 122 & 118 & 116 & 115 & 115 & 113 & 111 & 111 & 110 & 114 & 114 & 115 & 116 & 116 & 120 & 124 & 128 & 129 & 129 & 128 & - & - \\
\hline & $\mathrm{n}^{\circ}$ provincias & - & - & - & - & 3 & 3 & 3 & 3 & 3 & 3 & 3 & 3 & 3 & 4 & 4 & 6 & 6 & 8 & 9 & 9 & 9 & 10 & 12 & 12 & 12 & 12 & - & - \\
\hline \multirow{2}{*}{ Vitoria } & oficinas & 77 & 74 & 77 & 76 & - & - & - & - & - & - & - & - & - & - & - & - & - & - & - & - & - & - & - & - & - & - & - & - \\
\hline & $\mathrm{n}^{\circ}$ provincias & 2 & 1 & 2 & 3 & - & - & - & - & - & - & - & - & - & - & - & - & - & - & - & - & - & - & - & - & - & - & - & - \\
\hline \multirow{2}{*}{ Álava } & oficinas & 74 & 75 & 75 & 75 & - & - & - & - & - & - & - & - & - & - & - & - & - & - & - & - & - & - & - & - & - & - & - & - \\
\hline & $\mathrm{n}^{\circ}$ provincias & 2 & 3 & 3 & 3 & - & - & - & - & - & - & - & - & - & - & - & - & - & - & - & - & - & - & - & - & - & - & - & - \\
\hline \multirow{2}{*}{ Gip.-SS } & oficinas & - & - & - & - & 219 & 197 & 188 & 186 & 168 & 164 & 168 & 174 & 140 & 148 & 164 & 184 & 190 & 195 & 214 & 249 & 289 & 320 & 333 & 342 & 342 & 334 & - & - \\
\hline & $\mathrm{n}^{\circ}$ provincias & - & - & - & - & 5 & 5 & 5 & 5 & 4 & 4 & 4 & 4 & 4 & 4 & 4 & 8 & 12 & 13 & 18 & 24 & 30 & 32 & 32 & 33 & 33 & 33 & - & - \\
\hline \multirow[t]{2}{*}{ Guipúzcoa } & oficinas & 121 & 121 & 123 & 124 & - & - & - & - & - & - & - & - & - & - & - & - & - & - & - & - & - & - & - & - & - & - & - & - \\
\hline & $\mathrm{n}^{\circ}$ provincias & 4 & 4 & 4 & 4 & - & - & - & - & - & - & - & - & - & - & - & - & - & - & - & - & - & - & - & - & - & - & - & - \\
\hline \multirow{2}{*}{$\begin{array}{c}\text { San } \\
\text { Sebastián }\end{array}$} & oficinas & 91 & 93 & 93 & 93 & - & - & - & - & - & - & - & - & - & - & - & - & - & - & - & - & - & - & - & - & - & - & - & - \\
\hline & $\mathrm{n}^{\circ}$ provincias & 3 & 3 & 3 & 3 & - & - & - & - & - & - & - & - & - & - & - & - & - & - & - & - & - & - & - & - & - & - & - & - \\
\hline \multirow{2}{*}{ Caja Sur } & oficinas & 86 & 91 & 91 & 91 & 92 & 93 & 100 & 103 & 110 & 113 & 120 & 143 & 161 & 195 & 209 & 223 & 237 & 249 & 269 & 280 & 281 & 485 & 486 & 474 & 466 & 396 & 391 & 387 \\
\hline & $\mathrm{n}^{\circ}$ provincias & 9 & 9 & 9 & 9 & 9 & 9 & 9 & 9 & 9 & 9 & 9 & 11 & 11 & 11 & 11 & 12 & 14 & 15 & 15 & 15 & 15 & 16 & 16 & 16 & 16 & 14 & 14 & 10 \\
\hline \multirow{2}{*}{$\begin{array}{c}\text { Córdoba } \\
\text { pro. }\end{array}$} & oficinas & 108 & 109 & 111 & 111 & 110 & 115 & 116 & 118 & 120 & - & - & - & - & - & - & - & - & - & - & - & - & - & - & - & - & - & - & - \\
\hline & $\mathrm{n}^{\circ}$ provincias & 1 & 1 & 1 & 1 & 1 & 2 & 2 & 2 & 2 & - & - & - & - & - & - & - & - & - & - & - & - & - & - & - & - & - & - & - \\
\hline
\end{tabular}

\section{Fuente: Elaboración propia}

